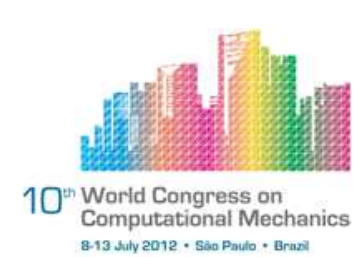

\title{
ON THE ELECTROMECHANICAL BEHAVIOR OF ELECTROLYTE SOLUTIONS
}

\author{
M. C. Reis ${ }^{1}$ and A. B. M. S. Bassi ${ }^{1}$ \\ ${ }^{1}$ Department of Physical Chemistry, Institute of Chemistry at the University of Campinas \\ (marreis@iqm.unicamp.br)
}

\begin{abstract}
Since the publication of Cosserat brothers' work, microcontinuum field theories have been making decisive progresses, concerning the fundamentals and the development of mathematical models, in several engineering and biomechanics topics. These theories, unlike the classical field ones, made possible to analyze many different properties of non-simple bodies. Thus, they attracted the researchers' attention, especially of those studying the transport of electrolytes in charged hydrated biological tissues. Nevertheless, some fundamental aspects of the electromechanical and chemical interactions are usually neglected. In fact, the study of ion-solvent and ion-ion interactions in electrolyte solutions is still an important research topic, because these interactions affect the drift of ions and the electric and chemical properties of solutions. Thus, in this work we developed a model for diluted electrolyte solutions using continuum thermodynamics. For reaching this aim, the continuum mixtures theory concepts were employed. Moreover, we supposed that the electrolyte solutions are material bodies with inner rigid structures (micropolar medium) which can interact with mechanical, quasi-static electromagnetic and chemical fields. Global balance laws for mass, linear and angular momenta, energy, and entropy, were settled and localized to obtain the local laws. From these balance laws, we distinguished the chemical behavior of two kinds of electrolytes, and emphasized the polar character of the solution, which is related to physical-chemical interactions between the ion and solvent molecules. In addition, we showed that translational and rotational movements contribute differently to the energy flux vector. While numerical results are not presented, the theoretical results obtained exhibit central importance for the discussion and prediction of physical-chemical phenomena in more complex materials, such as blood, biological tissues, polymeric suspensions, and slurries.
\end{abstract}

Keywords: Continuum mechanics, Micropolar continuum, Electrolyte solutions, Balance laws. 


\section{INTRODUCTION}

While most of the continuum mechanics treatises consider that the Cauchy's stress tensor is symmetric, it has long been known that anti-symmetric stress tensors may occur in continuum mechanics. The first to realize that some materials do not obey the constitutive law proposed by Cauchy, in which all torques are moments of forces, were the Cosserat brothers, in the beginning of the last century. They observed that many phenomena seem incompatible with classical continuum mechanics, which is based on the fundamental assumption that all balance laws are valid for every part of the body, and the state of the body at any material point is influenced by the neighborhood around that point exclusively. These assumptions make clear that classical continuum mechanics do not encompass the long-range effects of loads on the motion and evolution of the body.

Then, in order to include long-range effects on continuous bodies, Cosserat brothers proposed to describe these effects as functions of the direction, so that the body may be visualized as sets of points having vectors attached to them (directors). They considered these vectors as rigid triads, that is, material points were considered as geometrical points that posses properties similar to those of rigid particles (micropolar medium). But this approach soon revealed limited, due to the difficulties encountered to treat the material symmetry in constitutive equations [8]. In the second half of last century, more consistent polar theories were developed. Among these theories, there are Eringen's works [2, 3], where memory and diffusive effects, as well as electromagnetic interactions, were incorporated to the theory. Since then, polar continuum theories have been employed to study the thermomechanical behavior of blood, polymeric suspensions, crystal liquids, granular media etc. and, more recently, the properties of electrolyte solutions.

In this work, we propose a continuum model to study the thermomechanical behavior of electrolyte solutions. For this aim, we deduce the balance equations of the solution and its constituents, and we emphasize the resemblances and differences between this model and that for apolar continua. The proposed balance equations of mass and linear momentum have the same form for both polar and apolar continua. But the differences appear when the balance equations of angular momentum and energy are laid down. These differences come from phenomenological considerations on the physical model, related to additional quantities which are specific for polar continua. These quantities must be taken into account because the system behavior, as a whole, may not be described by macroscopic movements exclusively. Indeed, small velocity changes in volume elements, even though they are not macroscopically detectable, they do influence the system evolution. Thus, not only macroscopic movements should be analyzed, but also the movements that describe the internal evolution of the volume element. Hence, in order to validate the localization postulate, some additional kinematic variables must be introduced. Then, without recurring to a totally non-local theory, it is possible to evaluate the non-linear effects of electromagnetic fields generated at distant points, 
and movements of such points, on the point $\mathbf{x}$. Thus, the presented results apply to any polar mixture under the influence of electromagnetic fields.

\section{CONTINUUM MODEL PROPOSED}

The electrolyte solution will be considered to be a continuum mixture of polar constituents which may carry electric charges and/or electric dipole moments. The mixture as a whole is electrically neutral, but polarizable. Electromagnetic quantities will be considered under the quasi-electrostatic approximation because to study the electromechanical behavior of a mixture, both the balance equations of the classical theory of mixtures and the Maxwell equations are demanded.

In fact, it is well known that the Maxwell equations are invariant under Lorentz transformations, whereas the balance equations of continuum mechanics are invariant under Galilean transformations. But for the coherence of the continuum model proposed, it is required that the Maxwell equations invariant under the same kind of transformation for which the balance equations of classical mixture theory are invariant. This may be reached if just non relativistic velocities are considered and the system acoustic frequencies are lower than $10^{4} \mathrm{~Hz}$. Consequently, being known the sources of the electric and magnetic fields at a certain moment, at this time these fields are determined without concern for what the sources were any instant earlier [6].

All the results presented follow the formalism applied by Hutter and Johnk [4] and Truesdell [7], and all equations are written in their component forms by considering a Cartesian coordinate system. The direct notation is used throughout this paper. In particular, vectors in the Euclidean 3D-space are denoted by bold symbols and letters, while tensors are denoted by italic capital letters. The gradient and divergence with respect to spatial coordinates are indicated by $\nabla$ and div, respectively. A quantity corresponding to a particular material in the mixture is identified by placing a labeling index $a$ directly above the symbol for the quantity. Summations over the constituent indexes are always indicated by a summation sign, $\sum$, and the metric tensor and permutation symbols are respectively denoted by $e_{i j k}$ and $\epsilon_{i j k}$. This procedure is necessary in order to avoid confusion, since the usual notation employed in continuum mixture theories is frequently cumbersome.

In addition, all macroscopic fields are interpreted as averages of microscopic fields generated by the constituents, located on point $\mathbf{x}$ at time $t$. Thus, for example, $\mathbf{E}^{a}$ is the total electric field that acts over the species $a$ at coordinates $(\mathbf{x}, t)$, by considering the action of an externally applied electric field and electric fields generated from the interaction of $a$ with itself and other mixture constituents. 


\subsection{Mass balance}

Since we suppose a reactive continuum mixture, a mass production term, $c^{a}$, must be introduced in the mass balance equation. Then, we have the equation

$$
\frac{\partial \rho^{a}}{\partial t}+\operatorname{div}\left(\dot{\mathbf{x}}^{a} \rho^{a}\right)=c^{a}
$$

where $\rho^{a}$ is the mass density of constituent $a, \dot{\mathbf{x}}^{a}$ is the velocity of $a$, and the mass production

$c^{a}$ is given by $c^{a}=\sum_{j=1}^{n}\left(c^{j a}-c^{a j}\right)$, where $c^{a j}$ is the mass conversion velocity from constituent $a$ to constituent $j$ by volume unit, as a result of chemical reactions. If the constituent $a$ is converted into constituent $j$ at some point, but the constituent $j$ is not converted into constituent $a$ at the same point, then $c^{a j}>0$ and $c^{j a}=0$. Inversely, one has $c^{a j}=0$ and $c^{j a}>0$. Since chemical reactions can progress in both directions, $c^{a j}$ and $c^{j a}$ may be simultaneously positive at the same point. Because positive values for both $c^{a j}$ and $c^{j a}$ correspond to the direct and reverse velocities, $c^{a}$ may be positive, negative or null.

The introduction of terms related to the rate of mass conversion from a constituent into another is extremely important to associate the nature of the electrolyte to ion-ion interactions which, in turn, determine the properties of the electrolyte solution. How much these interactions affect the properties of the solution and how dense is the ionic distribution in the solution are equivalent questions. But the ionic distribution density depends on the nature of the electrolyte, that is, depends on whether it is a potential electrolyte or a real one.

Electrolytes that, through chemical reactions with solvent molecules, dissociate into ions are potential electrolytes. Most potential electrolyte solutions have only a small concentration of ions, so the effects of ion-ion interactions are frequently neglected. The behavior of these solutions is governed predominantly by the equilibrium position of the reaction between the potential electrolyte and the solvent molecules. Then, $c^{a j}$ and $c^{j a}$ are different for a same point of the mixture until the kinetic equilibrium is reached. In contrast, real electrolytes are those that originate ions in solution through physical interactions between the ions present in the ionic solid and the solvent molecules. In general, a real electrolyte is completely ionized when the solid is dissolved in water, so that the resulting solution consists only of solvated ions and solvent. For these solutions, the dependence of their properties on the concentration is determined by the interaction force between the ions.

The classification into real electrolyte and potential one is a modern classification that aims to describe the electrolyte behavior by its structure and not by its behavior in a particular solvent. However, the classification was historically made based on the electrolyte behavior in a specific solvent, usually water. Weak electrolytes were those that produce solutions with small electric conductivity when the electrolyte is dissolved, whereas strong electrolytes were those that originate solutions with high conductivity after dissolving the electrolyte. The disadvantage of this last classification is that, as soon as a solvent other than water is chosen, 
the electrolyte denominated a strong one in water can behave as a weak electrolyte in a nonaqueous solvent and vice-versa [1].

At each point, the mass balance equation for the mixture as a whole is obtained by considering null the sum of the mass production terms on all constituents that take part in the

chemical reactions, that is, $\sum_{a=1}^{n} c^{a}=0$. This expression imposes that the chemical reaction per se do not change the density at each point in the mixture. Thus, we have

$$
\frac{\partial \rho}{\partial t}+\operatorname{div}(\dot{\mathbf{x}} \rho)=0
$$

where the definition of mixture density was employed.

\subsection{Linear and angular momenta balances}

In the bulk of electrolyte solutions, where the gradient of the electric field is small, the ionic movement is the responsible for the conductive properties exhibited by the solution. This phenomenon, governed by the Ohm's law, implies the existence of some force whose character is not exclusively electrostatic. Indeed, ions in electrolyte solutions experiment mechanical and chemical forces, beyond the electrostatic forces that may be due to ion-ion and ion-dipole interactions. While coulombic forces are responsible for the ionic interactions, the polarization Kelvin's force is the result of the action of the electric field created by ions present in solution and/or an external source on the water molecules dipoles. Thus, the equation of linear momentum balance is

$$
\frac{\partial \rho^{a} \mathbf{g}^{a}}{\partial t}+\operatorname{div}\left(\rho^{a} \mathbf{g}^{a} \otimes \dot{\mathbf{x}}^{a}-T^{a}\right)=\rho^{a} \mathbf{b}^{a}+q^{a} \mathbf{E}^{a}+\mathbf{P}^{a} \cdot\left(\nabla \mathbf{E}^{a}\right)+\mathbf{m}^{a},
$$

where $\rho^{a} \mathbf{g}^{a}$ is the total linear momentum, by considering both mechanical and electromagnetic contributions, $T^{a}$ is the stress tensor, by considering both mechanical and electromagnetic contributions again, $\mathbf{b}^{a}$ is the mechanical body force, $q^{a}$ is the charge density, $\mathbf{E}^{a}$ and $\mathbf{P}^{a}$ are respectively the electric and polarization fields, and $\mathbf{m}^{a}$ is the linear momentum production. From Equation 3, the balance equation of linear momentum for the mixture is obtained by imposing that the sum of production terms on all constituents is null, according to the general postulate of continuum mixtures theory [7]. Then, it follows that

$$
\frac{\partial \rho \mathbf{g}}{\partial t}+\operatorname{div}(\rho \mathbf{g} \otimes \dot{\mathbf{x}}-T)=\rho \mathbf{b}+q \mathbf{E}+\mathbf{P} \cdot(\nabla \mathbf{E})
$$

where $\rho \mathbf{g}=\sum_{a=1}^{n} \rho^{a} \mathbf{g}^{a}, \rho \mathbf{b}=\sum_{a=1}^{n} \rho^{a} \mathbf{b}^{a}, q \mathbf{E}=\sum_{a=1}^{n} q^{a} \mathbf{E}^{a},[\mathbf{P} \cdot(\nabla \mathbf{E})]=\sum_{a=1}^{n}\left[\mathbf{P}^{a} \cdot\left(\nabla \mathbf{E}^{a}\right)\right]$, $T=\sum_{a=1}^{n} T^{a}-\sum_{a=1}^{n} \rho^{a} \mathbf{g}^{a} \otimes \mathbf{u}^{a}$ and the definition of diffusion velocity, $\mathbf{u}=\dot{\mathbf{x}}^{a}-\dot{\mathbf{x}}$, was employed. 
We have assumed that the constituents of the electrolyte solution are polar continua. Then, at every point of the mixture, and by considering every point as a punctual system, the total angular momentum of each constituent about an origin, $\mathbf{o}$, is given by the sum of two parts: the angular momentum of the punctual system about the position of its center of mass (spin), $\rho^{a} \mathbf{s}^{a}$, and the angular momentum of the punctual system center of mass about that origin, $\mathbf{o} \times \rho^{a} \mathbf{g}^{a}$. Analogously, the supply of angular momentum is given by the sum of the coupling of electromagnetic fields, $\mathbf{P}^{a} \times \mathbf{E}^{a}$, and the angular momentum, in relation to the origin, of the forces that act on the punctual system center of mass, $\mathbf{o} \times\left\{\rho^{a} \mathbf{b}^{a}+q^{a} \mathbf{E}^{a}+\left[\mathbf{P}^{a} \cdot\left(\nabla \mathbf{E}^{a}\right)\right]\right\}$. In turn, the flux of angular momentum is given by the coupling stresses, $C^{a}$, and $\mathbf{o} \times T^{a}$. Lastly, the angular momentum production is composed by the spin production, $\tau^{a}$, and the angular momentum production on the punctual system center of mass, $\mathbf{o} \times \mathbf{m}^{a}$. Once these quantities are defined, the balance equation of angular momentum is

$$
\begin{gathered}
\frac{\partial\left(\mathbf{o} \times \rho^{a} \mathbf{g}^{a}+\rho^{a} \mathbf{s}^{a}\right)}{\partial t}+\operatorname{div}\left[\left(\mathbf{o} \times \rho^{a} \mathbf{g}^{a}+\rho^{a} \mathbf{s}^{a}\right) \otimes \dot{\mathbf{x}}^{a}-\mathbf{o} \times T^{a}-C^{a}\right]= \\
\mathbf{P}^{a} \times \mathbf{E}^{a}+\mathbf{o} \times\left\{\rho^{a} \mathbf{b}^{a}+q^{a} \mathbf{E}^{a}+\left[\mathbf{P}^{a} \cdot\left(\nabla \mathbf{E}^{a}\right)\right]\right\}+\mathbf{o} \times \mathbf{m}^{a}+\boldsymbol{\tau}^{a} .
\end{gathered}
$$

Just like any tensor of second order, the stress tensor may be split up into a symmetric and a skew-symmetric part, that is, $T_{i j}^{a}=T_{(i j)}^{a}+T_{[i j]}^{a}$, where the brackets indicate anti-symmetry and the parenthesis, symmetry. By using the identity $\operatorname{div}\left(\mathbf{o} \times T^{a}\right)=\mathbf{o} \times \operatorname{div}\left(T^{a}\right)+\mathbf{t}^{\text {sks }}{ }^{a}$, where $\mathbf{t}^{s k s}{ }^{a}$ stands for the axial vector associated to $T_{[i j]}^{a}$, and the expression that converts local derivative into material derivative, Equation 5 becomes

$$
\rho^{a} \frac{\mathrm{d} \mathbf{s}^{a}}{\mathrm{~d} t}-\operatorname{div}\left(C^{a}\right)+\mathbf{t}^{s k s}{ }^{a}=\mathbf{P}^{a} \times \mathbf{E}^{a}+\boldsymbol{\tau}^{a}
$$

Equation 6 is known as the spin balance equation. This equation shows that the stress tensor associate to the stress vector of constituent $a$ is only symmetric if $\mathbf{s}^{a}$ is constant in time, the tensorial field $C^{a}$ is solenoidal, $\boldsymbol{\tau}^{a}=0, \mathbf{P}^{a}$ is aligned to $\mathbf{E}^{a}$. Moreover, Equation 6 allows to infer that the couplings due to electric fields play an important role in electrolyte solutions. For this purpose, impose that $\mathbf{s}^{a}$ is temporally constant, $C^{a}$ is a solenoidal field and $\boldsymbol{\tau}^{a}=0$. Thus, from Equation 6 follows that

$$
\mathbf{t}^{s k s}=-\mathbf{P}^{a} \times \mathbf{E}^{a} .
$$

However, $\mathbf{P} \times \mathbf{E}=e_{i j k} \mathrm{P}^{i} \mathrm{E}^{j} \mathbf{c}^{k}=e_{i j k} \mathrm{P}^{j} \mathrm{E}^{k} \mathbf{c}^{i}$ because $e_{i j k}=e_{j k i}$, being $e_{i j k}=\epsilon_{i j k}$ when the basis $\left(\mathbf{c}^{i}\right)_{i=1}^{3}$ is orthonormal. Further, as $\mathbf{t}^{s k s}=\epsilon_{i j k} T_{j k} \mathbf{c}^{i}$ for an orthonormal basis, one concludes that $T_{j k}=-\mathrm{P}_{[j} \mathrm{E}_{k]}$. Thus, when an electric field is imposed on an electrolyte solution, either by an external source or simply due to the presence of dissociated ions in solution, the equilibrium polarization of the solvent dipoles is affected. In fact, the solvent dipoles are rotated by a torque which aligns them to the electric field. The relaxation of this 
process to a new equilibrium position dissipates energy, generating a bigger friction on the ions than would occur if the solvent was a non-polarized viscous continuum. Thus, the ion mobility and solution conductivity are changed as a result of relaxation and electrophoretic effects, caused respectively by ion-ion interactions and viscous drag on the ion by the solvent.

The equation of angular momentum for the mixture as a whole is obtained by summing all the terms of Equation 5 on all constituents and employing the definition of diffusion velocity, $\mathbf{u}=\dot{\mathbf{x}}^{a}-\dot{\mathbf{x}}$. Then, we have

$$
\begin{gathered}
\frac{\partial(\mathbf{o} \times \rho \mathbf{g}+\rho \mathbf{s})}{\partial t}+\operatorname{div}[(\mathbf{o} \times \rho \mathbf{g}+\rho \mathbf{s}) \otimes \dot{\mathbf{x}}-\mathbf{o} \times T-C]= \\
\mathbf{P} \times \mathbf{E}+\mathbf{o} \times \rho \mathbf{b}+\mathbf{o} \times\{q \mathbf{E}+[\mathbf{P} \cdot(\nabla \mathbf{E})]\},
\end{gathered}
$$

because $\mathbf{o} \times \sum_{a=1}^{n} \mathbf{m}^{a}+\sum_{a=1}^{n} \boldsymbol{\tau}^{a}=\mathbf{0}$. As well as the stress tensor $T^{a}$, the tensor $T$ is not symmetric too, except if $\mathbf{s}$ is a constant, $C$ is a solenoidal field, and $\mathbf{P}$ and $\mathbf{E}$ are aligned. This indicates that the mixture does not behave as an apolar continuum body.

\subsection{Energy balance}

In the energy balance equation, the physical quantity to be balanced is the total energy density, composed by the kinetic, internal, and electromagnetic energy densities. Similarly, the flux, supply and production of energy are given by the thermodynamical and electromagnetic contributions. Thus,

$$
\begin{aligned}
& \frac{\partial\left(\rho^{a} \varepsilon^{a}+1 / 2 \rho^{a} \dot{\mathbf{x}}^{a}+1 / 2 \rho^{a} \mathbf{s}^{a} \cdot \boldsymbol{\omega}^{a}+1 / 2 \mathrm{E}^{a 2}\right)}{\partial t}= \\
& -\operatorname{div}\left[\left(\rho^{a} \varepsilon^{a}+1 / 2 \rho^{a} \dot{\mathbf{x}}^{a}+1 / 2 \rho^{a} \mathbf{s}^{a} \cdot \boldsymbol{\omega}^{a}+1 / 2 \mathrm{E}^{a 2}\right) \otimes \dot{\mathbf{x}}^{a}\right]+ \\
& +\operatorname{div}\left[\mathbf{S}^{a}+T^{a}\left(\dot{\mathbf{x}}^{a}\right)+C^{a}\left(\boldsymbol{\omega}^{a}\right)-\mathbf{h}^{a}\right]+\boldsymbol{\omega}^{a} \cdot\left(\mathbf{P}^{a} \times \mathbf{E}^{a}\right)+ \\
& +\mathbf{E}^{a} \cdot \frac{\partial \mathbf{P}^{a}}{\partial t}+\mathbf{E}^{a} \cdot \mathbf{i}^{a}+\rho^{a} r^{a}+\gamma^{a}+\dot{\mathbf{x}}^{a} \cdot\left\{\rho^{a} \mathbf{b}^{a}+q^{a} \mathbf{E}^{a}+\left[\mathbf{P}^{a} \cdot\left(\nabla \mathbf{E}^{a}\right)\right]\right\},
\end{aligned}
$$

where $\rho^{a} \varepsilon^{a}$ is the density of internal energy, $\boldsymbol{\omega}^{a}$ is the angular diffusion velocity, $\mathbf{h}^{a}$ is the energy flux vector, $\mathbf{S}^{a}$ is the Poyinting vector, $\boldsymbol{\omega}^{a}$ is the angular velocity, $\rho^{a} r^{a}$ is the energy supply due to mechanical forces, $\mathbf{i}^{a}$ is the migration current and $\gamma^{a}$ is the energy production. By using the expression for material derivative and Equations 2 and 3, the Equation 9 may be simplified to

$$
\begin{aligned}
& \frac{\mathrm{d}\left(\rho^{a} \varepsilon^{a}\right)}{\mathrm{d} t}+\operatorname{div}\left[\mathbf{h}^{a}-\mathbf{S}^{a}\right]= \\
& T^{a T} \cdot \nabla\left(\dot{\mathbf{x}}^{a}\right)+C^{a T} \cdot \nabla\left(\boldsymbol{\omega}^{a}\right)+\boldsymbol{\omega}^{a} \cdot T^{s k s}{ }^{a}+\mathbf{E}^{a} \cdot \frac{\partial \mathbf{P}^{a}}{\partial t}+\mathbf{E}^{a} \cdot \mathbf{i}^{a}+\rho^{a} r^{a}+\gamma^{a},
\end{aligned}
$$

where $T^{a T}$ and $C^{a T}$ denote respectively the transpose of $T^{a}$ and $C^{a}$. Equation 10 requires that, if the particles of the mixture are subject to deformations, this must not significantly 
change the angular velocity of the constituent, i.e., the mixture shows micropolarity.

Equation 10 is known as the balance equation of internal energy of the constituent. This equation differs from the balance equation of internal energy for apolar continuum bodies due to the presence of two terms, namely $C^{a T} \cdot \nabla\left(\boldsymbol{\omega}^{a}\right)$ and $\boldsymbol{\omega}^{a} \cdot T^{\text {sks }}{ }^{a}$. The first term, known as the energy from coupling stresses, is analogous to $T^{a T} \cdot \nabla\left(\dot{\mathbf{x}}^{a}\right)$ and represents the work per unit of time due to the stresses acting on a constituent. In turn, the second term is due to internal stresses resulting from interactions between the constituents, external agents or deformations of the mixture.

The balance equation of energy for the mixture as a whole is obtained by summing all the terms of Equation 9 on all constituents, and imposing that the total energy power generation is zero. Then, we have

$$
\begin{aligned}
& \frac{\partial\left(\rho \varepsilon+1 / 2 \rho \dot{\mathbf{x}}+1 / 2 \rho \mathbf{s} \cdot \boldsymbol{\omega}+1 / 2 \mathrm{E}^{2}\right)}{\partial t}= \\
& -\operatorname{div}\left[\left(\rho \varepsilon+1 / 2 \rho \dot{\mathbf{x}}+1 / 2 \rho \mathbf{s} \cdot \boldsymbol{\omega}+1 / 2 \mathrm{E}^{2}\right) \otimes \dot{\mathbf{x}}\right]+\operatorname{div}[\mathbf{S}+T(\dot{\mathbf{x}})+C(\boldsymbol{\omega})-\mathbf{h}]+ \\
& \boldsymbol{\omega} \cdot(\mathbf{P} \times \mathbf{E})+\mathbf{E} \cdot \frac{\partial \mathbf{P}}{\partial t}+\mathbf{E} \cdot \mathbf{i}+\rho r+\dot{\mathbf{x}} \cdot\{\rho \mathbf{b}+q \mathbf{E}+[\mathbf{P} \cdot(\nabla \mathbf{E})]\},
\end{aligned}
$$

where $\mathbf{h}=\sum_{a=1}^{n}\left[\mathbf{h}^{a}-\mathbf{S}^{a}-T^{a}\left(\mathbf{u}^{a}\right)-C^{a}\left(\boldsymbol{\omega}^{a}\right)+\left(\frac{1}{2} \rho^{a} \mathrm{u}^{a 2}+\frac{1}{2} \mathrm{E}^{a 2}+\frac{1}{2} \rho^{a} \varpi^{a 2}+\frac{1}{2} \rho^{a} \varepsilon^{a}\right) \mathbf{u}^{a}\right]$ is the energy flux vector, $\varpi$ is the translational diffusion velocity, and $\rho \varepsilon=\sum_{a=1}^{n}\left(\varrho^{a} \varepsilon^{a}+\frac{1}{2} \rho^{a} \dot{x}^{a 2}+\right.$ $\left.\frac{1}{2} \rho^{a} \varpi^{a 2}+\frac{1}{2} E^{a 2}\right)$, such as the total kinetic energy was split up into translational and rotational kinetic energies. Therefore, the expression for the energy flow vector of the mixture indicates that: (i) $\mathbf{h}$ can not be interpreted only as a heat flux vector, since it is composed by the heat flux vector, the power voltage and the coupling stresses of the diffusive motion and, also, the flow of convective energy associated to the diffusion and (ii) the translational and rotational movements contribute differently to the flow of energy in the mixture.

\subsection{Entropy balance}

There is an additive quantity named entropy for each constituent, so that the balance equation

$$
\frac{\partial}{\partial t} \rho^{a} \eta^{a}+\operatorname{div}\left(\rho^{a} \eta^{a} \dot{\mathbf{x}}^{a}+\phi^{a}\right)=\rho^{a} \sigma^{a}+\rho^{a} \pi^{a}
$$

is valid, where $\rho^{a} \sigma^{a}$ is the entropy supply, $\phi^{a}$ is the entropy flux, and $\pi^{a}$ is the entropy production. In turn, for the mixture as a whole

$$
\frac{\partial}{\partial t} \rho \eta+\operatorname{div}(\rho \eta \dot{\mathbf{x}}+\phi)=\rho \sigma+\rho \pi
$$


where $\sum_{a=1}^{n} \rho^{a} \eta^{a}=\rho \eta, \sum_{a=1}^{n} \rho^{a} \sigma^{a}=\rho \sigma, \sum_{a=1}^{n} \rho^{a} \pi^{a}=\rho \pi$, and $\sum_{a=1}^{n}\left(\phi^{a}+\rho^{a} \eta^{a} \mathbf{u}^{a}\right)=\phi$, such as $\eta^{a}$ and $\phi^{a}$ are objective constitutive quantities, as well as their respective sums. In this work, we have followed the dissipation axiom, proposed by Truesdell [7], which states that the entropy production for each constituent of the mixture need not be non-negative, even though the entropy production for the mixture must be $\rho \pi=\sum_{a=1}^{n} \rho^{a} \pi^{a} \geq 0$.

\section{CONCLUSION}

In this work, a thermodynamic model for dilute electrolyte solutions is proposed by considering the theory of mixtures in continuous media and electrodynamics. The polar and nonlocal nature of the mixture is highlighted, as well as the nonlinear composition of mechanical and electromagnetic theories. Moreover, balance equations of mass, linear and angular momenta, and energy and entropy, for the mixture as a whole and its constituents are presented. In the balance of angular momentum equation, terms are introduced for the spin in the supply, flow and production of angular momentum.

It is also shown that the stress tensor is not symmetrical, due to the polar nature of the constituents and mixture, and the influence of the electromagnetic fields and spin. By considering the energy balance equation for the mixture, it is clear that the vector $\mathbf{h}$ should not be interpreted as the heat flux vector of classical mechanics. Thus, the well known Clausius-Duhem inequality does not apply to polar mixtures and other entropy principles, for example, the Muller-Liu's principle [5], should be used to impose restrictions on the constitutive responses of the system.

\section{Acknowledgments}

The first author acknowledges the financial support from $\mathrm{CNPq}$ (the Brazilian National Council for Scientific and Technological Development).

\section{References}

[1] Bockris, J. O’M., Reddy, A.K.N., Modern Electrochemistry. Ionics, 2nd Ed., Kluwer Academic Publishers: New York, 2002.

[2] Eringen, A.C., Microcontinuum Field Theories. I. Foundations and Solids, Springer: New York, 1994.

[3] Eringen, A.C., Nonlocal Continuum Field Theories, Springer: New York, 2002.

[4] Hutter, K., Johnk, K.D., Methods of Physical Modeling. Continuum Mechanics, Dimensional Analysis, Turbulence, Springer: Berlin, 2004. 
[5] Liu, I-S., "Method of Lagrange Multipliers for Exploitation of the Entropy Principle", Arch. Ration. Mech. Anal. 46, 131-148, 1972.

[6] Melcher, J.R., Continuum Electromechanics, MIT Press: Cambridge, 1981.

[7] Truesdell, C.A., Rational Thermodynamics, Springer: New York, 1968.

[8] Truesdell, C.A., Noll, W., The Non-linear Field Theories of Mechanics, 3rd Ed., Springer: Berlin, 2004. 